\section{The accuracy of the Edinburgh visual loss diagnostic algorithm}

C Goudie ${ }^{1}$, A Khan ${ }^{1}$, C Lowe ${ }^{2}$ and M Wright ${ }^{1,2}$

\begin{abstract}
Purpose To assess the diagnostic accuracy of the Edinburgh visual loss algorithm.

Methods This was a prospective study. Patients referred to the Edinburgh Eye Pavilion with visual loss were assessed using the Edinburgh Visual Loss Algorithm by either a medical student, an inexperienced ophthalmology trainee or an optometrist in the Lothian Optometry Treat and Teach clinic. Accuracy of this 'algorithmassisted' diagnosis was then compared with the 'gold-standard' diagnosis, made by an experienced ophthalmologist. Accuracy of the pre-algorithm diagnosis, made by the referrer, was also compared with the algorithm-assisted diagnosis. Results All patients referred with visual loss were eligible for inclusion. Seventy patients were assessed; two were excluded. Pre-algorithm accuracy of referral of patients with visual loss was $51 \%(30 / 59)$. Overall, the algorithm-assisted diagnosis was correct $84 \%(57 / 68)$ of the time. The algorithm correctly diagnosed: retina in $71 \%$ of cases (5/7), macula in $86 \%(25 / 29)$, peripheral retina in $100 \%(2 / 2)$, optic nerve in $71 \%(5 / 7)$, media opacity in $89 \%(16 / 18)$, post chiasmal in $100 \%$ $(4 / 4)$, and refractive error in $0 \%(0 / 1)$.

Accuracy of diagnosis was similar for each algorithm user; medical student $81 \%$, inexperienced ophthalmology trainee $84 \%$ and optometrist $92 \%$.

Discussion The baseline diagnostic accuracy of clinicians who are inexperienced in ophthalmology rose from 51 to $84 \%$ when patients were assessed using the algorithm. This algorithm significantly improves the diagnostic accuracy of referrals to the hospital eye service, regardless of the user's previous ophthalmic experience. We hope we have demonstrated its potential as a learning tool for inexperienced clinicians.

Eye (2015) 29, 1483-1488; doi:10.1038/eye.2015.146; published online 21 August 2015
\end{abstract}

\section{Introduction}

This is the second in a series of three studies assessing the accuracy of diagnostic algorithms for each of the three most commonly encountered ophthalmic presentations: red eye (s), ${ }^{1}$ visual loss, and diplopia. As discussed in the previous paper, ${ }^{1}$ our three algorithms highlight those key aspects of both the history and the examination which allow the inexperienced examiner to come to the likely diagnosis. Algorithms are, therefore, simply the user-friendly version of the diagnostic and/or treatment thought processes of more experienced clinicians.

Algorithms are being used more and more in medicine; junior doctors are being asked to provide cross cover over a greater range of specialties than before. Current examples in healthcare include diagnosing pulmonary embolus, ${ }^{2}$ differentiating types of tachycardia ${ }^{3}$ and a number of algorithms published by the Resuscitation Council (UK) such as Basic and Advanced Life Support. ${ }^{4}$ Diagnostic algorithms have been suggested to reduce time, effort and bias, ${ }^{5}$ and cost of care and errors. ${ }^{6}$ It has been been introduced, experienced healthcare workers continue to demonstrate autonomy. ${ }^{6}$

The causes of visual loss range from benign; refractive error, to life threatening; posterior visual pathway lesions. Most patients are initially seen in primary care, by GPs, A\&E doctors or optometrists who have varying levels of experience in dealing with acute ophthalmic patients. Unfortunately, most UK doctors including GPs and A\&E doctors have had between 0 and 2 weeks ophthalmology teaching, ${ }^{7}$ during their undergraduate training, leaving them inexperienced and wary of dealing with acute eye problems. ${ }^{8}$ In many A\&E departments nurse practitioners are taking on more responsibility and independently managing patients, without any formal eye shown that in situations where algorithms have
${ }^{1}$ Department of Ophthalmology, Princess Alexandra Eye Pavilion, Edinburgh, Scotland

${ }^{2}$ College of Medicine and Veterinary Medicine, University of Edinburgh, Edinburgh, Scotland

Correspondence: M Wright, Department of Ophthalmology, Princess Alexandra Eye Pavilion, Chalmers Street, Edinburgh EH3 9HA, UK

Tel: +44(0) 131 5363778; Fax: +44(0) 1315363897 E-mail: mark.wright@luht. scot.nhs.uk

Received: 19 April 2015 Accepted in revised form: 12 July 2015 Published online: 21 August 2015 
training or experience. Optometrists are becoming increasingly involved in ophthalmic primary care in the community and are the first port of call for many patients with an eye emergency problem, particularly in Scotland. ${ }^{9}$ These groups of inexperienced clinicians (with respect to acute ophthalmological problems), including junior ophthalmologists, may benefit from simple clinical algorithms. Algorithms are always a compromise between having enough detail to cover the most commonly encountered diagnoses whilst remaining simple enough to use.

The aim of this prospective study was to assess the diagnostic accuracy of the Edinburgh Visual Loss Algorithm, published in 'Ophthalmology Pocket Tutor', 10 which was created by Dr Mark Wright, Consultant Ophthalmologist and Lead for Undergraduate Ophthalmology Education at Edinburgh University.

\section{Materials and methods}

Patients presenting to the Edinburgh Eye Pavilion with visual loss, usually having been referred from primary care (optometry or general practice) or less commonly from secondary care (non ophthalmology hospital setting) were assessed using the Edinburgh Visual Loss Algorithm (Figure 1) by one of three groups;

- One fourth year medical student (CL; following the week-long clinical attachment in ophthalmology).

- Junior ophthalmology trainees ( $<2$ years of ophthalmology experience).

- Optometrists (based in LOTT; Lothian Optometry Treat \& Teach clinic).

This data was collected in order to assess the accuracy of the 'algorithm-assisted' diagnosis. An experienced ophthalmologist, defined as having a minimum of 2 years full-time training, then independently examined the patient to arrive at a 'gold-standard' diagnosis. The degree of concordance between the algorithm-assisted diagnosis and the gold standard was then assessed.

The source and diagnosis of each referral from primary care or another hospital specialty was also noted to estimate the 'pre-algorithm' diagnostic accuracy.

Ethical approval was granted through the University of Edinburgh College of Medicine, in conjunction with the South East Scotland Research Ethics Service.

\section{Results}

Seventy completed forms were returned during the recruitment period of February to December 2014. Two were excluded; both were filled in incorrectly by the algorithm user, leaving 68 cases available for analysis.

\section{Profile of referrer}

The breakdown of referrer by type was; Optician 62\%, GP $12 \%$, A+E Doctor 4\%, doctors from other hospital specialties $4 \%$, nurse practitioners in minor injuries units $3 \%$ and diabetic retinopathy screeners $2 \%$. Six percent of patients self-presented without a referral and referral information was unavailable in $7 \%$.

\section{Pre-algorithm accuracy of referrals with visual loss}

Out of these 68 cases, a 'pre-algorithm' referral diagnosis was unavailable in 9; in 5 the initial referral was unavailable or lost and 4 patients self-presented to the acute eye clinic without being referred from the community. This left 59 cases which allowed a direct comparison to be made between the diagnoses made by non-experts with and without the visual loss algorithm. In 16 of the 59 cases the referrer simply described the symptoms and signs but did not include a diagnosis. For the purposes of the analysis these referrals were classified as incorrect diagnoses. This was the case for referrals from; community optometrist in 5 out of 42 patients, by doctors and nurse practitioners in 10 out of 14 patients and by diabetic retinopathy screeners in 1 out of 1 patient. The overall pre-algorithm accuracy of referral of patients with visual loss was 51\% (30/59). Optometrists were the most accurate referrers making the correct diagnosis $67 \%$ $(28 / 42)$ of the time. GPs were correct in $13 \%(1 / 8)$ of referrals, A\&E doctors in 33\% (1/3). There were no correct referral diagnoses from; doctors from other hospital specialties $(0 / 3)$, nurse practitioners in minor injuries units $(0 / 2)$, and diabetic retinopathy screeners $(0 / 1)$.

Of these 59 cases, no attempt at the diagnosis was made by the community optometrist in 5 out of 42 patients, by doctors and nurse practitioners in 10 out of 14 patients and by diabetic retinopathy screeners in 1 out of 1 patient. For the purposes of the analysis these were classified as incorrect diagnoses.

The pre-algorithm accuracy of referral of patients with visual loss was 51\% (30/59). Optometrists were the most accurate referrers making the correct diagnosis $67 \%$ $(28 / 42)$ of the time. GPs were correct in $13 \%(1 / 8)$ of referrals, A\&E doctors in 33\% (1/3), and doctors from other hospital specialties were correct in $0 \%(0 / 3)$. Nurse practitioners in minor injuries units were correct in $0 \%$ $(0 / 2)$ of referrals and diabetic retinopathy screeners referred one patient without knowing the diagnosis $0 \%(0 / 1)$. 


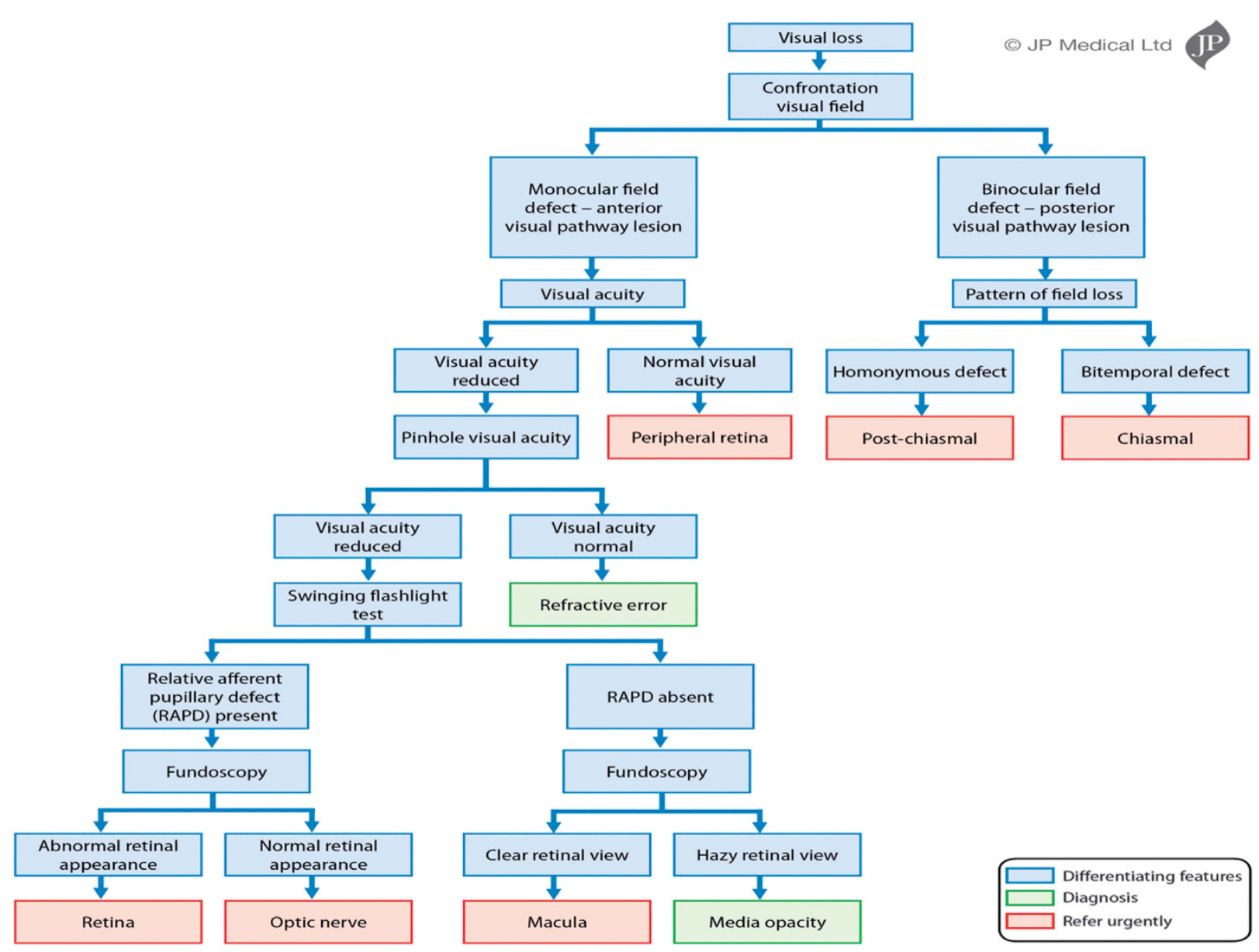

Figure 1 The Edinburgh Visual Loss Diagnostic Algorithm.

\section{Profile of algorithm user}

The breakdown of algorithm user by type was; medical student $45 \%$, optometrist $18 \%$, and junior ophthalmology trainee $37 \%$.

\section{Diagnostic accuracy of the algorithm-assisted diagnoses}

Overall, the algorithm-assisted diagnosis was correct $84 \%$ $(57 / 68)$ of the time. The 'gold-standard' ophthalmologists made seven out a possible eight diagnoses contained within the algorithm; there were no 'chiasmal' diagnoses. The algorithm correctly diagnosed: retina in $71 \%$ of cases (5/7), macula in $86 \%(25 / 29)$, peripheral retina in $100 \%(2 / 2)$, optic nerve in $71 \%(5 / 7)$, media opacity in $89 \%(16 / 18)$, post chiasmal in $100 \%(4 / 4)$, and refractive error in $0 \%(0 / 1)$; see Figure 2. The accuracy of diagnosis was broadly similar for each algorithm user; medical student $81 \%$, ophthalmology trainee (ST2 and below) $84 \%$ and optometrist $92 \%$.

Sensitivity and specificity of the algorithm for the different diagnoses can be seen in Figure 3.

\section{Analysis of the incorrect algorithm-assisted diagnoses}

The algorithm-assisted diagnosis was incorrect in 16\%

$(11 / 68)$ of the cases. The two sources of diagnostic error were either defects in the algorithm itself; $9 \%(6 / 68)$ or failure of the inexperienced clinician to elicit a clinical sign; $7 \%(5 / 68)$.

Six incorrect diagnoses were made due to problems with the algorithm Three of these errors were due to dual/preexisting pathology. One patient in whom the dominant cause of their visual loss was macula (wet age-related macular degeneration AMD) also had cataract resulting in a hazy retinal view and consequently an incorrect algorithm-assisted diagnosis of media opacity. Another patient with new wet AMD had an incorrect algorithmassisted diagnosis of a post-chiasmal cause of visual loss due to a bilateral, although central, visual field defect secondary to longstanding bilateral dry AMD. The last patient in this group had dual pathology; new onset media opacity caused by idiopathic vitreous haemorrhage and pre-existing non-arteritic anterior ischaemic optic neuropathy (NAION). This patient was incorrectly 
diagnosed as 'retina', because of a relative afferent pupillary defect (RAPD) related to the NAION.

Two patients were classified incorrectly due to the absence of an RAPD despite extensive retinal pathology. One of these patients had a macula-off retinal detachment and the other had a non-ischaemic central retinal vein occlusion (CRVO).

The last patient in this group had a dislocated intraocular lens implant, causing a large refractive error that was not fully corrected by a pin hole. As a result this was diagnosed incorrectly as a media opacity.

Five incorrect diagnoses were made due to failure to elicit a clinical sign Two patients were wrongly diagnosed because the

- Correctly diagnosed by algorithm $\square$ Incorrectly dignosed by algorithm

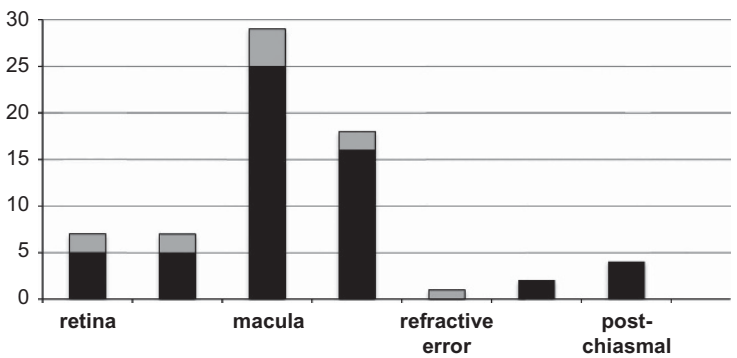

Figure 2 Graph to show the number of patients with each eye condition and the accuracy of the Edinburgh Visual Loss Algorithm in correctly diagnosing these patients. non-specialist did not detect an RAPD resulting in a diagnosis of macula or media opacity, when the goldstandard diagnosis was optic nerve. In one case an RAPD was falsely detected by the non-specialist, the patient had wet AMD. In two cases an error occurred when the non-specialist incorrectly determined whether there was a clear or hazy retinal view on fundoscopy resulting in one incorrect diagnosis each of media opacity and macula.

\section{Comparison of diagnoses made by non-experts with and without the algorithm}

In the 59 cases where a 'pre-algorithm' referral diagnosis was available, diagnostic accuracy improved from 51 to $86 \%$ when using the algorithm. This is shown, grouped by referral source, in Table 1.

\section{Discussion}

The baseline (pre-algorithm) diagnostic accuracy in this study from all referrers was 51\%. The only other study that provided figures on referrals from GPs and ophthalmic opticians specifically relating to visual loss found marginally higher diagnostic accuracies, ranging from $51-72 \%$ depending on the source of referral. ${ }^{11}$ These figures demonstrate a need for a diagnostic aid when nonophthalmologists assess patients who present with visual

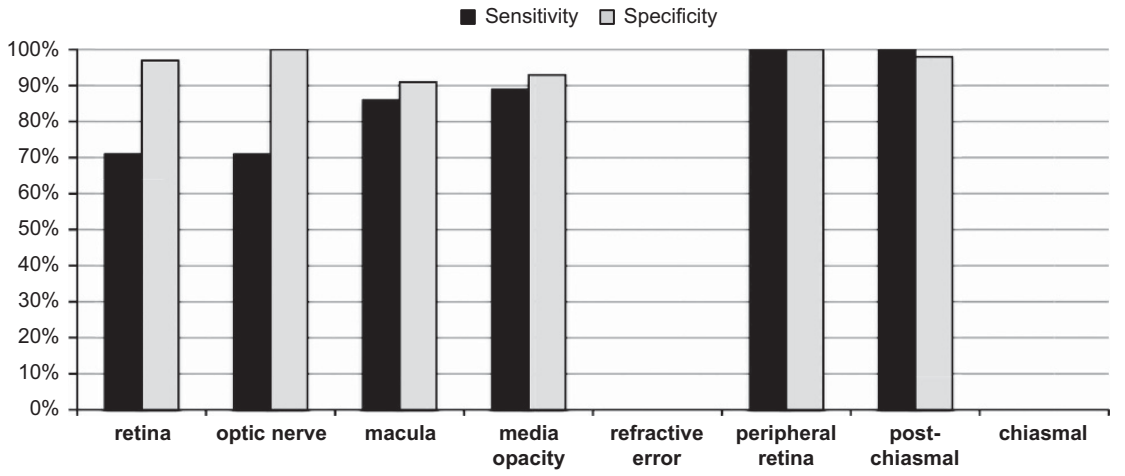

Figure 3 Graph to show the sensitivity and specificity of the Edinburgh Visual Loss Algorithm for each diagnosis.

Table 1 Table showing the pre-algorithm accuracy of each referral (grouped by referral source) compared with diagnosis made in the same patient by a non-expert using the Edinburgh Visual Loss Algorithm

\begin{tabular}{lcc}
\hline Source of referral & Pre-algorithm accuracy of diagnosis & Accuracy when non-expert uses the algorithm in same patient \\
\hline Optometrist & $67 \%(28 / 42)$ & $95 \%(40 / 42)$ \\
GP & $13 \%(1 / 8)$ & $50 \%(4 / 8)$ \\
A\&E & $33 \%(1 / 3)$ & $100 \%(3 / 3)$ \\
Hospital doctors & $0 \%(0 / 3)$ & $67 \%(2 / 3)$ \\
Minor injuries unit nurse practitioner & $0 \%(0 / 2)$ & $50 \%(1 / 2)$ \\
Diabetic retinopathy screener & $0 \%(0 / 1)$ & $100 \%(1 / 1)$ \\
Total & $51 \%(30 / 59)$ & $86 \%(51 / 59)$
\end{tabular}

Nine patients were seen with no referral diagnosis available.

Visual loss algorithm-assisted diagnosis was correct in $6(67 \%)$ of these patients. 
loss. Our study showed an overall algorithm-assisted diagnostic accuracy of $84 \%$. Its simplicity of use is confirmed by our finding that the most inexperienced user; a medical student with only $14 \mathrm{~h}$ of undergraduate ophthalmology teaching, demonstrated a very high level of diagnostic accuracy; $81 \%$.

The ideal triage system facilitates the patient being assessed by the most appropriate person at the first visit. The algorithm correctly diagnosed the presence of a posterior visual pathway lesion ('post-chiasmal') in 100\% $(4 / 4)$ patients. The most appropriate triage decision for this group of four patients may have been to organize neuro-imaging prior to the first ophthalmic consultation or indeed redirection to a neurovascular clinic if a vascular cause was thought likely.

The ideal algorithm should allow the user to demonstrate very high sensitivity with respect to potentially the most serious conditions; posterior visual pathway lesions, post chiasmal in $100 \%(4 / 4)$, and also to have high levels of specificity for the more minor conditions; media opacity in 89\% (16/18).

The incorrect identification of RAPDs was involved in 5 out of 11 wrong diagnoses. RAPDs can be difficult to elicit, particularly for less experienced examiners. ${ }^{12}$ In order to improve inexperienced examiners skill in eliciting an RAPD we have demonstrated this clinical technique at https://www.eemec.med.ed.ac.uk/pages/resources / mw-ophthalmology-page. Only one error resulted from difficulty of an inexperienced examiner using a direct ophthalmoscope. The difficulty in performing accurate direct ophthalmoscopy by different specialties has been described elsewhere. ${ }^{13}$ In our study this difficulty contributed to a diagnosis of 'don't know' as seen in 11 out of 18 referrals from doctors and nurses. This was taken into account when designing the algorithm by simplifying fundoscopy findings, for example, retinal appearance either 'clear' or 'hazy'.

In reaching an algorithm assisted diagnosis of a retinal problem we have assumed that both the peripheral and central retina (macula) are involved. Significant retinal pathology with an estimated $30-50 \%$ of nerve axons need to damaged before an RAPD is evident. In our study two patients with 'retinal' pathology did not have an RAPD. One of these had a retinal detachment and the other had a non-ischaemic CRVO, whilst both these patients had involvement of the peripheral and central retina neither had significant retinal ischaemia and therefore no accompanying RAPD.

The algorithm failed to reach the correct anatomical diagnosis in three cases when there was dual/pre-existing eye disease. Deciding which of two pathologies, for example, cataract and AMD is the dominant one relies upon clinical experience and confirms the limitations of our algorithm where more complex decision making is required.
During the course of the study a problem relating to the urgency of referrals in patients with media opacity became evident. The most common cause of media opacity is cataract, which causes visual loss of gradual onset and can be referred routinely as per the algorithm. However, vitreous haemorrhage also results in media opacity but typically with sudden onset and always requires prompt assessment. We will update the algorithm to reflect this.

One of the educational benefits to the inexperienced clinician of using the algorithm when assessing patients who present with visual loss is that it acts as a clinical prompt reminding the novice of each of the pertinent components of the examination and the ideal sequence in which to perform them.

The main limitation of the study was the lack of patients with a diagnosis of 'chiasmal' disease. This is a very infrequently encountered scenario and hence difficult to recruit patients to.

\section{Conclusion}

This is the first time the diagnostic accuracy of any visual loss algorithm has been assessed. The baseline diagnostic accuracy of non-ophthalmologists rose from 51 to $84 \%$ when patients were assessed using The Edinburgh Visual Loss Diagnostic Algorithm. This algorithm significantly improves the diagnostic accuracy of referrals to the hospital eye service, regardless of the user's previous ophthalmic experience. We hope we have demonstrated its potential as a learning tool for inexperienced clinicians. A number of open access learning tools including downloadable copies of five diagnostic algorithms and narrated lectures accompanying the algorithms are available at https:/ /www.eemec.med.ed. ac.uk/pages/resources/mw-ophthalmology-page.

\section{Summary}

\section{What was known before}

- Visual loss is a common presenting complaint in patients attending $\mathrm{A}+\mathrm{E}$, optometrists, and GPs and can be the presenting symptom of a life threatening disease. To date, there are no validated diagnostic algorithms to help inexperienced clinicians come to the correct diagnosis, which would aid hospital triage. Previous studies show low rates of diagnostic accuracy and low confidence among inexperienced clinicians when assessing patients with visual loss.

What this study adds

- The baseline diagnostic accuracy of non-ophthalmologists; optometrists, hospital doctors and GPs was 51\%, thus it reinforced the need for a diagnostic aid.

- The overall diagnostic accuracy of the Edinburgh Diplopia Algorithm is $84 \%$, with minimal differences in diagnostic accuracy when used by clinicians of widely varying experience. The diagnostic improvement resulting from the use of the algorithm should result in more accurate triage of patients referred to the hospital eye service. 


\section{Conflict of interest}

MW has received minimal royalties from the sales of 'Ophthalmology Pocket Tutor' where the algorithm has been published. The remaining authors declare no conflict of interest.

\section{References}

1 Timlin H, Butler L, Wright M. The accuracy of the Edinburgh Red Eye Diagnostic Algorithm. Eye 2015; 29(5): 619-624.

2 Lau EW, Ng GA. Comparison of the performance of three diagnostic algorithms for regular broad complex tachycardia in practical application. Pacing Clin Electrophysiol 2002; 25(5): 822-827.

3 Shih HC, Wen YS, Ko TJ, Wu JK, Su CH, Lee CH. Noninvasive evaluation of blunt abdominal trauma: prospective study using diagnostic algorithms to minimize nontherapeutic laparotomy. World J Surg 1999; 23(3): 265-269.

4 Resuscitation Council (UK) Algorithms for Guidelines 2010. Available at: http://www.resus.org.uk/pages/Public.htm (accessed on 10 August 2015).

5 Ranjan D, Loewenstein D, Greig M, Acevedo A, Potter E, Appel J et al. Reliability and validity of an algorithm for the diagnosis of normal cognition, mild cognitive impairment, and dementia: implications for multicentre research studies. Am J Geriatr Psychiatry 2010; 18: 363-370.

6 Christensen-Szalanski JJ, Diehr PH, Wood RW, Tompkins RK. Phased trial of a proven algorithm at a new primary care clinic. Am J Public Health 1982; 72: 16-21.

7 Welch S, Eckstein M. Ophthalmology teaching in medical schools: a survey in the UK. Br J Ophthalmol 2011; 95: 748-749.

8 Featherstone PI, James C, Hall MS, Williams A. General practitioners' confidence in diagnosing and managing eye conditions: a survey in south Devon. Br J Gen Pract 1992; 42: 21-24.

9 NHS Scotland. About NES Optometry. Available at: http:/ / www.nes.scot.nhs.uk/education-and-training/bydiscipline/optometry/about-nes-optometry.aspx (accessed on 10 August 2015).

10 Borooah S, Wright M, Dhillon B. Ophthalmology Pocket Tutor. JP Medical Ltd: London, 2012.

11 Harrison RJ, Wild JM, Hobbley AJ. Referral patters to ophthalmic outpatient clinics by general practitioners and ophthalmic opticians and the role of these professionals in screening for ocular disease. BMJ 1988; 297(6657): 1162-1167.

12 Lankaranian D, Altangerel U, Speath GL, Leavitt JA, Steinmann WC. The usefulness of a new method of testing for a relative afferent pupillary defect in patients with ocular hypertension and glaucoma. Trans Am Ophthalmol Soc 2005; 103: 200-208.

13 Benbassat J, Polak BC, Javitt JC. Objectives of teaching direct ophthalmoscopy to medical students. Acta Ophthalmol 2012; 90(6): 503-507. 